\title{
A UNIFIED MODEL FOR THE RADIO AND OPTICAL IMAGES AND SPECTRA OF NOVA V1974 CYG 1992
}

\author{
R. M. HJELLMING \\ National Radio Astronomy Observatory ${ }^{\dagger}$ \\ Socorro, NM 87801-0387, USA
}

\begin{abstract}
A model for nova shells which is a combination of wind model and spherically symmetric, Hubble flow shell model, fits the light curve, image, and nebular line shape data for Nova V1974 Cyg 1992. The results show that after outburst the shell is ejected by a wind-like outflow with a linear velocity gradient, and that after $180 \mathrm{~d}$ this outflow stops. The shape of the elliptical inner boundary at this time determines the subsequent emission measure structure seen in images, light curves, and nebular line profiles.
\end{abstract}

\section{Introduction}

The first classical novae to be observed during a major portion of their radio light curves were HR Del 1967, FH Ser 1970, and V1500 Cyg 1975 (Hjellming et al. 1988). Two models that have been used to fit the data for these and other novae: a variable wind model (Kwok 1983) where the ejecta are continuous with a constant velocity and a mass loss rate which is proportional to the inverse of the time since outburst; and a Hubble flow shell model (Hjellming et al. 1988) where a finite shell is ejected with a velocity gradient proportional to the radius. Both models assumed spherical symmetry. In this paper we discuss the modifications that are needed to fit the images and spectral line shapes of Nova V1974 Cyg 1992.

Until now models used to interpret radio light curves have assumed spherical symmetry. For the variable wind model (Kwok 1983) a constant velocity ( $v_{\text {wind }}$ ) wind with a mass loss rate inversely proportional to the time since outburst $\left(t-t_{0}\right)$ gives a density profile in the nova shell described by $\left.\rho(t)=\dot{M}\left(t_{0}\right) /\left[\left(t / t_{0}-1\right) v_{\text {wind }} 4 \pi \mu R^{2}\right)\right]$, where $\mathrm{R}$ is the radius, $\mu$ is the mean

\footnotetext{
${ }^{\dagger}$ The National Radio Astronomy Observatory is operated by Associated Universities, Inc. under a cooperative agreement with the National Science Foundation.
} 

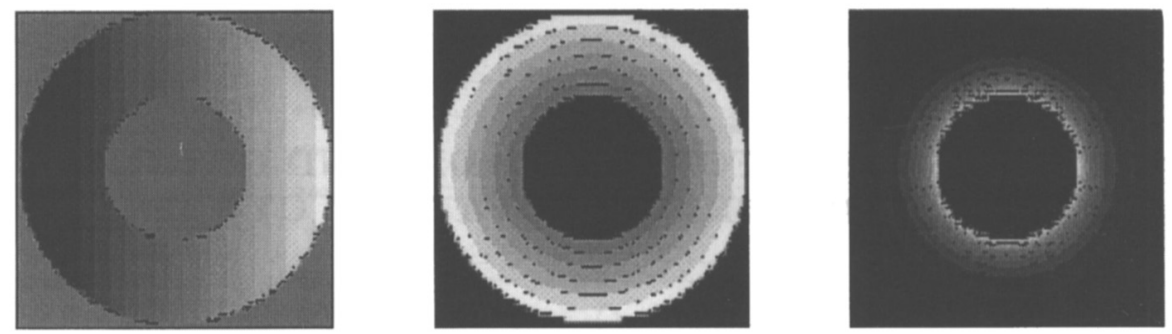

Figure 1. Images of the internal structure of a shell with a linear velocity gradient showing: (left) radial velocities as seen by an observer to the left of the page; (centre) a cross-section of the velocity struction in the shell, where the velocities are proportional to radius; and (right) the emission measure image of the shell as seen from any direction.

molecular weight, and $\dot{M}\left(t_{0}\right)$ is the initial mass loss rate. For the Hubble flow shell model (Hjellming et al. 1988) a finite shell is ejected with inner and outer radii $R_{1}=v_{1}\left(t-t_{0}\right)+R_{10}$ and $R_{2}=v_{2}\left(t-t_{0}\right)+R_{20}$, where $v_{2} \geq v_{1}$, and inside the shell $v \propto R$, so $\rho(t)=M_{\text {shell }} /\left[4 \pi \mu R^{2}\left(R_{2}(t)-R_{1}(t)\right)\right]$. Both models give density profiles that are inversely proportional to both $\left(t-t_{0}\right)$ and $R^{2}$, so they mostly have the same properties. However, for the pure wind model a finite inner radius never develops and there is no velocity gradient across the shell; the lack of finite inner radius at later times implies that a completely optically thin, detached shell does not develop, and the late decay curves do not match the observations (Hjellming 1990, 1995).

For a spherically symmetric nova shell with a linear velocity gradient and a finite inner radius, the radial velocity, velocity (or radius), and emission measure structure of the shell is shown in Fig. 1. These figures indicate why the observable properties of optically thin shells are largely determined by the mass near the inner shell boundary. If one combines the emission measure and radial velocity structure to predict a line profile, there is only one nebular line shape predicted by these models: $\phi(v)=$ $\left\{\left[v_{1}(t) v_{2}\right] /\left\{4\left[v_{1}(t)-v_{2}\right]\right\}\right\}\left[v^{-2}-v_{2}^{-2}\right]$ for $v_{1}(t) \leq|v| \leq v_{2}$, and $\phi(v)=\phi\left[v_{1}(t)\right]$ for $|v| \leq v_{1}$. While $v_{2}$ is a constant, $v_{1}(t)$ can vary until shell ejection stops and the inner boundary is formed.

Another important point relates to the generally higher masses derived from radio light curves or images (Hjellming 1995). Part of the reason for this is obvious from Fig. 1, because while the shell has equal mass in equal increments of radius, the four inverse powers of radius determining the contributions to the emission measure mean that any observations of the optically thin shell will be dominated by the innermost part of the shell. 


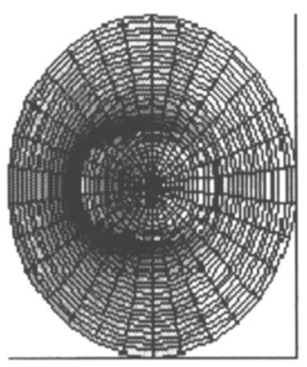

Figure 2. A ruled surface display for a model of the optically depth structure of Nova V1974 Cyg 1992 at $313 \mathrm{~d}$ after outburst, showing the different elliptical inner and outer boundaries; it should be rotated counterclockwise by $25^{\circ}$ to match the position angle of the nova shell.

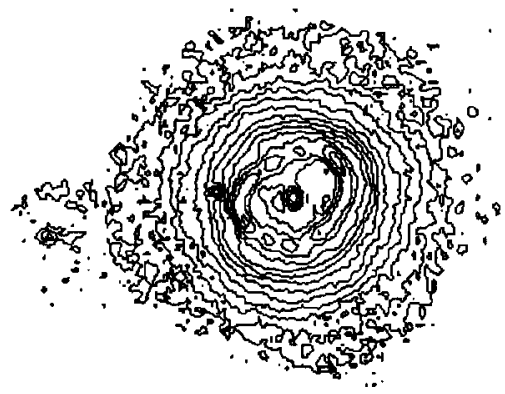

Figure 3. A contour display of the 1994 January 9 HST image of Nova V1974 Cyg 1992, where adjacent contour levels differ by a factor of 1.58 . The major axis of the brightest part of the inner ellipse is $0.38^{\prime \prime}$.

\section{Observations and models for Nova V1974 Cyg 1992}

As discussed by Hjellming (1995) the extensive Merlin (Pavelin et al. 1993), VLA, and HST images of V1974 Cyg 1992 make it necessary to adopt models which deviate from spherical symmetry, and it was proposed that a modification of the spherical shell model, where the inner and outer boundaries are allowed to be different ellipses, would solve the problem of the early shape appearing elliptical with a major axis at position angle $25^{\circ}$, while the late VLA and HST images show a roughly elliptical shape with a position angle of $115^{\circ}$. Computations of the radiative transfer through shells with linear velocity gradients and different ellipsoidal inner and outer boundaries have been carried out. Fig. 2 shows a ruled surface display of the optical depth $313 \mathrm{~d}$ after outburst for a model that fits the radio light curves and produces roughly the right shapes for the optically thick and optically thin states. In this model a spherically symmetric shell with inner and outer apparent angular velocities of 0.08 and $0.2^{\prime \prime} \mathrm{yr}^{-1}$ is replaced by ellipsoids with triaxial angular velocities of $0.07,0.06$, and $0.09^{\prime \prime} \mathrm{yr}^{-1}$ for the inner 
boundary and $0.18,0.18$, and $0.23^{\prime \prime} \mathrm{yr}^{-1}$ for the outer boundary. where the middle number is the line of sight component. The remaining parameters of the model are $M_{\text {shell }}=3.110^{-4} \mathrm{M}_{\odot}$ for a mean molecular weight of 1.273 , a distance of $2 \mathrm{kpc}$, and temperature that increases linearly with time from about $5000 \mathrm{~K}$ at $40 \mathrm{~d}$ after outburst to about $26000 \mathrm{~K}$ at $300 \mathrm{~d}$ after outburst, as discussed by Hjellming (1995). However, one additional factor must be included in the modeling to fit all the infrared, millimetre and centimetre light curves, in addition to the changes in nebular line profiles. One must assume that the inner shell regions remain near the surface of the white dwarf until about $180 \mathrm{~d}$ after outburst, and at that time shell ejection effectively ceases and the above-mentioned inner shell parameters become valid. This same elliptical boundary model then correctly predicts nebular line profiles that change from the early, flat topped (except for fluctuations due to clumps) line shapes seen in 1992 September to the narrower line shapes seen in 1993 April (Shore et al. 1993).

While Fig. 2 is an approximate fit to the needed radio shapes of V1974 Cyg 1992 (Hjellming 1995), there are two other indications that the ellipsoidal boundary model is roughly correct. Fig. 3 is a contour display of the 1994 January 9 HST image (obtained from the STScI/HST archive) of this nova, in the form where adjacent contour levels differ by a factor of 1.58. The roughly even spacing of the contours indicates that the brightness distribution is a power law, and is more extended than the bright inner part of the shell. Most of the outer contours are elliptical with a position angle consistent with $25^{\circ}$, and there is a gradual transition from to roughly spherially symmetric, and then to the innermost, brightest portions which are ellipses with a position angle consistent with $115^{\circ}$.

\section{References}

Hjellming, R.M., 1990, in "Physics of Classical Novae", eds Cassatella, A., Viotti, R., Springer-Verlag, New York, p169

Hjellming, R.M., 1995, in "Cataclysmic Variables", eds A. Bianchini et al., Kluwer, p139 Hjellming, R.M., Wade, C.M., Vandenberg, N.R., Newell, R.T., 1988, AJ, 84, 1619

Kwok, S, 1983, MNRAS, 202, 1149

Pavelin, S., Davis, R.J., Morrision, L.V., et al., 1993, Nature, 363, 424

Shore, S.N., Sonneborn, G., Starrfield, S., et al., 1993, AJ, 106, 2408 